\title{
Cancer Drugs Fund requires further reform
}

\author{
Reliance on "real world" observational data undermines evidence base for clinical practice
}

\author{
Richard Grieve professor ${ }^{1}$, Keith Abrams professor ${ }^{2}$, Karl Claxton professor ${ }^{3}$, Ben Goldacre senior \\ clinical research fellow ${ }^{4}$, Nicholas James professor ${ }^{5}$, Jon Nicholl professor ${ }^{6}$, Mahesh Parmar \\ professor $^{7}$, Chris Parker consultant oncologist ${ }^{8}$, Jasjeet S Sekhon professor ${ }^{9}$, Liam Smeeth \\ professor $^{10}$, David Spiegelhalter professor ${ }^{11}$, Mark Sculpher professor ${ }^{3}$
}

${ }^{1}$ Department of Health Services Research and Policy, London School of Hygiene and Tropical Medicine, London WC1H 9SH, UK; ${ }^{2}$ Department of Health Sciences, University of Leicester, Leicester, UK; ${ }^{3}$ Centre for Health Economics, University of York, York, UK; ${ }^{4} \mathrm{Centre}$ for Evidence-Based Medicine, University of Oxford, Oxford, UK; ${ }^{5}$ University Hospitals Birmingham NHS Foundation Trust, Birmingham, UK; ${ }^{6}$ School of Health and Related Research, University of Sheffield, Sheffield, UK; ${ }^{7}$ Medical Research Council Clinical Trials Unit, University College London, London, UK; ${ }^{8}$ Institute of Cancer Research: Royal Cancer Hospital, London, UK; ${ }^{9}$ Travers Department of Political Science, Department of Statistics, University of California, Berkeley, CA, USA; ${ }^{10}$ Department of Non-Communicable Disease Epidemiology, London School of Hygiene and Tropical Medicine; ${ }^{11}$ Statistical Laboratory, Centre for Mathematical Sciences, Cambridge, UK

The reforms to the Cancer Drugs Fund implemented in July were an excellent opportunity to generate evidence on the effectiveness of new cancer drugs. ${ }^{1}$ Unlike under the previous arrangements, data on patients' outcomes will have to be collected for all drugs funded by the scheme. However, the reforms' stated reliance on "real world" (observational) data will not generate reliable evidence of effectiveness. We propose an alternative model, using timely randomised controlled trials within routinely collected data sources, to establish which drugs are relatively effective. The current arrangement encourages early access to drugs, with high prices but uncertain benefits, whereas our proposal will provide high quality evidence for future decisions and therefore larger gains in population health. The Cancer Drugs Fund was introduced in England in 2010-11 with a $£ 50 \mathrm{~m}$ ( $€ 58 \mathrm{~m} ; \$ 65 \mathrm{~m}$ ) budget, which grew to $£ 340 \mathrm{~m}$ in 2015-16 without evidence of patient benefits. ${ }^{2}$ Since the reforms, the National Institute for Health and Care Excellence (NICE) is responsible for appraising all new cancer drugs, and the fund will pay for those drugs which have a chance of being judged cost effective, after two years of "real world" data collection. However, once a drug is recommended, precedent suggests it is difficult to stop its use. For example, NHS funding for $\beta$ interferon for patients with multiple sclerosis continued after an independent evaluation that used "real world" data found it did not improve patient outcomes. ${ }^{3}$

The current reforms to the fund will not encourage manufacturers to conduct randomised trials, and NICE will have to make decisions without trial evidence. The central role given to "real world" data is a major cause for concern. Accurate estimates of relative effectiveness require that outcomes are compared for patients who do, and do not, take the new drug, but who have similar prognostic characteristics. ${ }^{4}$ However, in observational studies key characteristics are unmeasured and estimates of effectiveness are biased by residual confounding. Also, the conduct of observational studies is more prone to manipulation by those with vested interests than in randomised trials. The presumption that, without addressing these difficulties, "real world" data can provide unbiased evidence, ignores all we know about good research design for identifying causal effects, and the reasons why well designed randomised trials are the cornerstone of evidence based medicine. ${ }^{5}$

Instead, we propose that NICE makes "only in research" recommendations, whereby these drugs are available only within pragmatic, low cost, randomised trials. These studies should be designed to provide timely, unbiased estimates of effectiveness by routinely randomising patients to the new drug or current practice at the point of NHS care. ${ }^{6}$ This can be achieved only with strong support from funders, ethics committees, regulators, and central government, and if a research culture is embedded within the NHS. ${ }^{7}$ These trials require clinician time to recruit patients and investment in informatics, ${ }^{6}$ but the costs will be low compared with the drug fund's budget. Furthermore, follow-up data can be collected from the UK's high quality, routinely collected clinical datasets (including the world's largest cancer registry) and linked to existing radiotherapy and chemotherapy datasets, such as the Systemic Anti-Cancer Therapy dataset, ${ }^{8}$ and to sources of electronic health records, such as the Clinical Practice Research Datalink. Future trials could be linked to cohorts with genetic information. 
The trial designs can be flexible and provide a platform for new drugs as they emerge. Multi-arm, multistage trials, in particular, allow more treatments to be assessed than traditional two arm trials, and enable the range of patient subgroups and treatments to adapt as the data provide insights about which patients respond best to which drugs. ${ }^{9}$ These trials can recruit patients from within observational databases and include international centres to improve statistical power and generalisability.

For some new cancer drugs, an NHS funded randomised trial may provide insufficient additional value to justify the costs. ${ }^{10}$ If there is an ongoing trial for regulatory purposes it may be more efficient to delay a NICE decision pending evidence on long term outcomes from the regulatory trial. For drugs for which a trial is judged unethical or impractical, careful non-randomised studies should be conducted to minimise confounding, by collecting longitudinal data on all relevant prognostic characteristics and outcomes for patients receiving and not receiving the new drug. To reduce residual confounding further, studies should collect data on characteristics that predict treatment selection but are unrelated to outcomes. ${ }^{11}$

The reforms to the Cancer Drugs Fund, related initiatives such as the accelerated access review, ${ }^{12}$ and comparative effectiveness research using, for example, Surveillance, Epidemiology and End Results (SEER) data linked to Medicare, ${ }^{13}$ all rely on "real world" data. These initiatives will undermine the evidence base for clinical practice; once these products are widely used, randomisation will be impossible. Instead, we propose that expensive new agents are available only within rapid, flexible, and efficient randomised trials. Building NHS capacity for this programme would capitalise on the UK's strength in trials, generate long term evidence of value worldwide, and yield large benefits to patients.

We thank Anirban Basu, John Cairns, Manuel Gomes, Neil Hawkins, Miguel Hernan, Noemi Krief, Alec Miners, Stephen O'Neill, and Bernard Rachet for helpful discussions.

Competing interests: We have read and understood BMJ policy on declaration of interests and declare the following interests: RG, KA, KC,
CP, LS, JN, and MS have done consultancy or advisory board work for various drug companies. KA conducts research funded by Janssen; MP and JN have multiple educational or research grants from drug companies. RG, KA, and JN have undertaken consultancy for the NICE scientific advisory service, and MS undertakes NICE technology assessment reviews. BG receives income from speaking and writing for lay audiences on problems in science and medicine.

Provenance and peer review: Not commissioned, peer reviewed.

1 Mayor S. New "managed access" process for Cancer Drugs Fund to go ahead, NHS England confirms. BMJ 2016;352:i1208. doi:10.1136/bmj.i1208 pmid:26926879.

2 Claxton K. Pharmaceutical pricing: early access, the Cancer Drugs Fund and the role of NICE. Centre of Health Economics, University of York, 2016. http://www.york.ac.uk/media/ che/documents/policybriefing/Drug_prices.pdf

$3 \mathrm{McCabe} \mathrm{C}$, Chilcott J, Claxton K, et al. Continuing the multiple sclerosis risk sharing scheme is unjustified. BMJ 2010;340:c1786. doi:10.1136/bmj.c1786. pmid:20522655.

4 Sekhon JS, Grieve RD. A matching method for improving covariate balance in cost-effectiveness analyses. Health Econ 2012;21:695-714. doi:10.1002/hec.1748 pmid: 21633989.

5 Cochrane AF. Effectiveness and efficiency: random reflections on health services. Taylor and Francis, 1972.

6 van Staa TP, Dyson L, McCann G, et al. The opportunities and challenges of pragmatic point-of-care randomised trials using routinely collected electronic records: evaluations of two exemplar trials. Health Technol Assess 2014;18:1-146. doi:10.3310/hta18430. pmid: 25011568.

7 Pollard A, Reiner A, John T, et al. Expediting clinical trials in a pandemic. Electronic reponse to de Jong et al. The future of influenza. BMJ 2009. http://www.bmj.com/rapidresponse/2011/11/02/expediting-clinical-trials-pandemic

8 Mestre-Ferrandiz J, Towse A, Dellamano R, Pistollato M. Multi-indication pricing: pros, cons and applicability to the UK. Office of Health Economics, 2015. https://www.ohe.org/ publications/multi-indication-pricing-pros-cons-and-applicability-uk

9 Parmar MK, Carpenter J, Sydes MR. More multiarm randomised trials of superiority are needed. Lancet 2014;384:283-4. doi:10.1016/S0140-6736(14)61122-3 pmid:25066148.

10 Claxton K, Palmer S, Longworth L, et al. A comprehensive algorithm for approval of health technologies with, without or only in research; the key principles for informing coverage decisions. Value Health 2016 [Epub ahead of print]. doi:10.1016/j.jval.2016.03.2003.

11 Angrist JD, Imbens GW, Rubin DB. Identification and causal effects using instrumental variables. J Am Stat Assoc 1996;91:444-55. doi:10.1080/01621459.1996.10476902.

12 Department for Business, Innovation and Skills, Department of Health. Accelerated access review. Interim report. Review of innovative medicines and medical technologies, supported by Wellcome Trust. 2015. https://www.gov.uk/government/uploads/system/uploads/ attachment_data/file/471562/AAR_Interim_Report_acc.pdf

13 National Institute of Health. National Cancer Institute. Division of Cancer Control and Population Sciences. Health care delivery research program, SEER-Medicare linked database, 2016. http://healthcaredelivery.cancer.gov/seermedicare/

Published by the BMJ Publishing Group Limited. For permission to use (where not already granted under a licence) please go to http://group.bmj.com/group/rights-licensing/ permissions 